\title{
Absence of Singular Continuous Spectrum for Certain Self-Adjoint Operators
}

\author{
E. Mourre \\ Centre de Physique Théorique, CNRS Marseille, F-13288 Marseille Cedex 2, France
}
Abstract. We give a sufficient condition for a self-adjoint operator to have the following properties in a neighborhood of a point $E$ of its spectrum:
a) its point spectrum is finite;
b) its singular continuous spectrum is empty;
c) its resolvent satisfies a class of a priori estimates.

\section{Notations, Definitions, and Main Theorem}

Let $H$ be a self-adjoint operator on a Hilbert space $\mathscr{H}$. We will denote by $\mathscr{H}_{n}(n \in \mathbf{Z})$ the Hilbert space constructed from the spectral representation for $H$ with the scalar product:

$$
(\Phi \mid \Psi)_{n}=\int\left(\lambda^{2}+1\right)^{n / 2}\left(\Phi \mid P_{H}(\lambda \lambda) \Psi\right) .
$$

For functions $P \in L^{\infty}(\mathbf{R}), P_{H}$ will denote the associated operator given by the usual functional calculus.

$P_{H}(E, \delta)$ will denote the spectral projection for $H$ onto the interval $(E-\delta, E+\delta)$. $P_{H}^{p}$ and $P_{H}^{c}$ will denote the spectral projectors respectively onto the point spectrum and the continuous spectrum of $H ; \sigma_{c}(H)=\mathbf{R} /\{E \in \mathbf{R} \mid E$ is an eigenvalue of $H\}$.

If $A$ is a self-adjoint operator and $D(A) \cap D(H)$ is dense in $\mathscr{H}, i[H, A]$ will denote the symmetric form on $D(A) \cap D(H)$ given by

$$
(\Phi \mid i[H, A] \Psi)=i\{(H \Phi \mid A \Psi)-(A \Phi \mid H \Psi)\}
$$

for $\Psi, \Phi \in D(A) \cap D(H)$. If this form is bounded below and closeable, $i[H, A]^{0}$ will denote the self-adjoint operator associated to the closure [1].

1. Definition. Let $H$ be a self-adjoint operator on a Hilbert space with domain $D(H)$; a self-adjoint operator $A$ is a conjugate operator for $H$ at a point $E \in \mathbf{R}$ if and only if the following conditions hold:

(a) $D(A) \cap D(H)$ is a core for $H$.

(b) $e^{+i A \alpha}$ leaves the domain of $H$ invariant and for each $\Psi \in D(H)$

$$
\sup _{|\alpha|<1}\left\|H e^{+i A \alpha} \Psi\right\|<\infty \text {. }
$$


(c) The form $i[H, A]=i(H A-A H)$ defined on $D(A) \cap D(H)$ is bounded below and closeable; moreover, the self-adjoint operator $i[H, A]^{0}$ associated to its closure admits a domain containing $D(H)$.

(d) The form defined on $D(A) \cap D(H)$ by $\left[[H, A]^{0}, A\right]$ is bounded as a map from $\mathscr{H}_{+2}$ into $\mathscr{H}_{-2}$.

(e) There exist strictly positive numbers $\alpha$ and $\delta$ and a compact operator $K$ on $\mathscr{H}$, so that:

$$
P_{H}(E, \delta) i[H, A]^{0} P_{H}(E, \delta) \geqq \alpha P_{H}(E, \delta)+P_{H}(E, \delta) K P_{H}(E, \delta) .
$$

Theorem. Let $H$ be a self-adjoint operator, having a conjugate operator $A$ at the point $E \in \mathbf{R}$, (i.e. suppose $H$ and $A$ satisfy conditions (a)-(e) above). Then there is a neighborhood $(E-\delta, E+\delta)$ of $E$ so that:

1. In $(E-\delta, E+\delta)$ the point spectrum of $H$ is finite.

2. For each closed interval $[a, b] \subset(E-\delta, E+\delta) \cap \sigma_{c}(H)$, there exists a finite constant $c_{0}$ so that:

$$
\sup _{\substack{\operatorname{Re} z \in[a, b] \\ \operatorname{Im} z \neq 0}}\left\||A+i|^{-1}(H-z)^{-1}|A+i|^{-1}\right\| \leqq c_{0} .
$$

Remark. The above theorem gives a method for obtaining a priori estimates of Agmon type [2] for certain self-adjoint operators, following from the existence of the conjugate operator $A$ of $H$ in the neighborhood of some point.

The essential condition in the definition of conjugate operator is condition (e); the other conditions justify the algebraic manipulations. To obtain the a priori estimates on $(H-z)^{-1}$ when $z$ approaches a point $E \in \sigma_{c}(H)$, we prove a priori estimates, uniform in $\varepsilon$ and $z$, on the operator $\left(H-z-i \varepsilon B^{*} B\right)^{-1}$. Here $\varepsilon$ and $\operatorname{Im} z$ have the same sign, $\operatorname{Re} z \in\left(E-\delta_{0}, E+\delta_{0}\right)$, and $B^{*} B=P_{H}\left(E, 2 \delta_{0}\right) i[H, A] P_{H}\left(E, 2 \delta_{0}\right)$. This estimate is obtained by proving a differential inequality of the form:

$$
\left\|\frac{d}{d \varepsilon} F_{z}(\varepsilon)\right\| \leqq K\left(\varepsilon,\left\|F_{z}(\varepsilon)\right\|\right)
$$

for $F_{z}(\varepsilon)=|A+i|^{-1}\left(H-z-i \varepsilon B^{*} B\right)^{-1}|A+i|^{-1}$.

In Sect. I, we give examples and applications. As new results we obtain the absence of singular continuous spectrum and a priori estimates in the following two cases:

(a) Relatively compact perturbations of certain pseudo-differential operators.

(b) Three-body Schrödinger operators with long-range two-body forces.

In Sect. II we give the proof of the main theorem.

\section{Examples and Applications}

\section{The Laplacian}

Let $\mathscr{H}=L^{2}\left(\mathbf{R}^{n}, d^{n} x\right), H=H_{0}=-\Delta$ and

$$
A=\frac{1}{4}(x \cdot p+p \cdot x) \quad p=-i \nabla .
$$

$A$ is the generator of the dilations introduced by Combes and used in [3]. - $\triangle$ and $A$ are defined on $\mathscr{S}$, the $\mathscr{C}^{\infty}$ functions of rapid decrease. $\mathscr{S}$ is a core for 
$H$. The explicit formula:

$$
e^{+i A \alpha}\left(H_{0}+i\right)^{-1}=\left(e^{-\alpha} H_{0}+i\right)^{-1} e^{+i A \alpha}
$$

shows that $e^{+i A \alpha}$ eqave $D(H)$ invariant. $\mathscr{S}$ is invariant under the dilation group and $i[-\Delta, A]=-\Delta$ in the sense of quadratic forms on $\mathscr{S}$. By Proposition II.1, condition (c) holds on $D(A) \cap D(H)$ and $i[H, A]^{\circ}=-\Delta$. Condition (d) then reduces to condition (c). Condition (e) is trivially satisfied at any point $E \neq 0$ by choosing $\delta<\frac{|E|}{2}$.

\section{Two-Body Schrödinger Operators}

Let

$$
\mathscr{H}=L^{2}\left(\mathbf{R}^{n}, d^{n} x\right), \quad H=-\Delta+V .
$$

We will often write $H_{0}$ for $-\Delta$. Much work has been done on these operators and we refer the reader to [4] for detailed references. Moreover, recently a very intuitive method has been introduced by Enss to prove asymptotic completeness for such systems [5].

We shall suppose that:

(i) $V$ is $H_{0}$ compact;

(ii) the operator $i\left\{V \frac{x p+p x}{4}-\frac{x p+p x}{4} V\right\}$ is defined on $\mathscr{S}$ and coincides on $\mathscr{S}$ with an $H_{0}$ compact operator $B$.

(iii) $B$ admits a decomposition: $B=B_{s}+B_{l}$ where $B_{s}^{*}|x|$ and $|x| B_{s}$ are $H_{0}$ bounded operators, and $\left[B_{l}, x p+p x\right]$ coincides on $\mathscr{S}$ with a form coming from an $H_{0}$ compact operator.

Remark. When $V$ is the operator of multiplication by a function $v(x),[V, x p+p x]$ $=2 i x \cdot \nabla v$, so that condition (ii) is satisfied if $x \cdot \nabla v$ is $H_{0}$ compact. Condition (iii) is satisfied if there is a smooth function $j(x)$ of compact support such that the operators $x_{i} \frac{\partial}{\partial x_{i}}\left\{(1-j(x)) x_{j} \frac{\partial v}{\partial x_{j}}\right\}$ are $H_{0}$ compact for all $i, j$.

Theorem I.1. If $V$ is a symmetric operator satisfying hypotheses (i)...(iii), then the operator $(\operatorname{sgn} E) A$ is conjugate to $H=H_{0}+V$ at all $E \neq 0 .\left(A=\frac{1}{4}(x p+p x)\right.$.)

If $E<0$, then 0 and $\mathbb{1}$ are also conjugate operators to $H$ at $E$.

Proof. Since $V$ is $H_{0}$ compact, $D(H)=D\left(H_{0}\right)$. By Example 1, $D\left(H_{0}\right)$ and therefore $D(H)$ is left invariant by $e^{+i A \alpha}$. By hypothesis (ii) the form $i[H, A]$ coincides on $\mathscr{S}$ with the form associated to the symmetric operator $H_{0}+B$ on $\mathscr{S}$, hence by Proposition II.1, condition (c) holds with $i[H, A]^{0}=H_{0}+B$.

To show that condition (d) holds, we write:

$$
\left[A, i[H, A]^{0}\right]=\left[A, B_{s}\right]+\left[A, H_{0}+B_{l}\right]
$$

the first term is bounded as a map from $\mathscr{H}_{+2}$ into $\mathscr{H}_{-2}$ by hypotheses (iii), the second coincides on $\mathscr{S}$ with the quadratic form of an $H_{0}$ bounded, self-adjoint operator. 
Let us verify condition (e).

$$
P_{H}(E, \delta) i[H, A]^{0} P_{H}(E, \delta)=P_{H}(E, \delta)\{H-V+B\} P_{H}(E, \delta) .
$$

Since $V$ and $B=i[V, A]$ are $H$ compact operators, by taking $\delta<\frac{|E|}{2}$ we have, letting $P_{H}(E, \delta)=P_{H}$,

$$
P_{H} i[H, A]^{0} P_{H} \geqq \frac{E}{2} P_{H}+P_{H} K P_{H} \quad \text { if } \quad E>0 .
$$

If $E$ is negative, we can see that the following two relations hold

$$
\begin{aligned}
P_{H} i[H,-A]^{0} P_{H} & \geqq \frac{|E|}{2} P_{H}+P_{H}-K P_{H} \\
P_{H} i[H, A]^{0} P_{H} & =P_{H}\left(H_{0}+B\right) P_{H} .
\end{aligned}
$$

Adding them, we see that 0 and therefore $\mathbb{1}$ are both conjugate operators for $H$ at energy $E<0$.

Remarks. As a consequence of Theorem I.1, we proved that the eigenvalues of $H$ can only accumulate at $E=0$, and are of finite multiplicity; outsided of them, the resolvent $(\mathrm{H}-\mathrm{z})^{-1}$ satisfies a priori estimate of Agmon's type [2].

\section{Perturbations of Pseudo-Differential Operators}

In [6], among the extensions of the method introduced in [5], the author proves similar results for short-range perturbations of pseudo-differential operators.

Let $\mathscr{H}=L^{2}\left(\mathbf{R}^{n}, d^{n} x\right)$ and denote by $L^{2}\left(\mathbf{R}^{n}, d^{n} p\right)$ the Hilbert space obtained by Fourier transformation.

Let $h_{0}(p)$ be a measurable function from $R^{n}$ to $R$ and $h_{0}$ the associated multiplication operator on $L^{2}\left(\mathbf{R}^{n}, d^{n} p\right)$. Suppose that:

$$
\lim _{|p| \rightarrow \infty}\left|h_{0}(p)\right|=\infty \text {. }
$$

Definition. $E \in \mathbf{R}$ is a regular point of $h_{0}$ if and only if there is a neighborhood $\left(E-\delta_{0}, E+\delta_{0}\right)$ of $E$ so that on

$$
O\left(E, \delta_{0}\right)=\left\{p \in \mathbf{R}^{n}|| h_{0}(p)-E \mid<\delta_{0}\right\} .
$$

$h_{0}$ is $\mathscr{C}^{m}$ for an $m \geqq 3$ and

$$
\sum_{i=1}^{n}\left(\frac{\partial h_{0}}{\partial p_{i}}\right)^{2}(p) \geqq \alpha>0, \quad p \in O\left(E, \delta_{0}\right) .
$$

Definition. $h_{0}+V$ is a regular perturbation of $h_{0}$ if $V$ satisfies the following conditions. 
1. $V$ is a symmetric $h_{0}$-compact operator.

2. For all real valued $g \in \mathscr{C}_{0}^{m}\left(\mathbf{R}^{n}\right)$, the $\mathscr{C}^{m}$ functions of compact support, the operators

$$
B_{i}=\left(x_{i} g(p)+g(p) x_{i}\right) V-V\left(x_{i} g(p)+g(p) x_{i}\right)
$$

are defined on $\mathscr{S}$ and extended to bounded, $h_{0}$-compact operators.

3. $\left[x_{j} g(p)+g(p) x_{j}, B_{i}\right]$ is bounded as a map from $\mathscr{H}_{+2}$ to $\mathscr{H}_{-2}$.

Theorem I.2. Let $H=h_{0}+V$ be a regular perturbation of $h_{0}$. For each regular point $E$ of $h_{0}$, there is an operator $A$ conjugate to $H$ at $E$.

Corollary I.3. Let $h_{0}+V$ be a regular perturbation of $h_{0}$. For each regular point $E$ of $h_{0}$, there is a neighborhood $(E-\delta, E+\delta)$ so that

1. the point spectrum of $h_{0}+V$ is finite in $(E-\delta, E+\delta)$.

2. For all $[a, b] \subset(E-\delta, E+\delta) \cap \sigma_{c}(H)$ there is a finite constant $c_{0}$ so that:

$$
\sup _{\substack{\operatorname{Re} z \in[a, b] \\ \operatorname{Im} z \neq 0}}\left\|(1+|x|)^{-1}(H-z)^{-1}(1+|x|)^{-1}\right\| \leqq c_{0} .
$$

Proof. Since $\left|h_{0}(p)\right| \rightarrow \infty$ as $|p| \rightarrow \infty, O\left(E, \delta_{0}\right)$ is a bounded subset of $\mathbf{R}^{n}$, so that we can find a $\mathscr{C}^{m-1}$ vector field $g_{i}(p) i \in\{1, \ldots, n\}$ of compact support in $\mathbf{R}^{n}$, with

$$
\begin{array}{r}
g_{i}(p)=\frac{\partial h_{0}}{\partial p_{i}}(p) \text { if } \quad p \in O\left(E, \delta_{0}\right) \\
g_{i}(p)=0 \text { if } \quad\left|h_{0}(p)\right|>M_{0} .
\end{array}
$$

Let $\hat{A}$ the formally symmetric operator defined on $L^{2}\left(\mathbf{R}^{n}, d^{n} p\right)$ by

$$
\hat{A}=\sum_{i=1}^{n} g_{i}(p) i \frac{\partial}{\partial p_{i}}+\frac{i}{2} \frac{\partial g_{i}}{\partial p_{i}}(p)=\frac{1}{2} \sum_{i}\left(g_{i} x_{i}+x_{i} g_{i}\right)
$$

By the commutator theorem [4] it is easily seen that $\hat{A}$ is essentially self-adjoint on the domain of $x^{2}=\sum_{i=1}^{n} x_{i}^{2}$.

Let $A$ be the self-adjoint extension so obtained. Since $D\left(x^{2}\right) \cap D\left(h_{0}\right)$ is a core for $h_{0}, D(A) \cap D\left(h_{0}\right)$ is a core for $h_{0}$. One can easily see (cf. Appendix A.1) that the unitary group $e^{+i A \alpha}$ is actually the group of unitary transformations on $L^{2}\left(\mathbf{R}^{n}, d^{n} p\right)$ associated with the group of diffeomorphisms $\Gamma_{\alpha}: \mathbf{R}^{n} \mapsto \mathbf{R}^{n}$ determined by the differential equation:

$$
\begin{aligned}
\frac{d}{d \alpha} \Gamma_{\alpha}^{i}(p) & =g_{i}\left(\Gamma_{\alpha}(p)\right) \\
\Gamma_{0}(p) & =p .
\end{aligned}
$$

It follows that $e^{+i A \alpha}$ leaves invariant the functions $\Psi(p)$ with support contained in $\left\{p \in \mathbf{R}^{n}|| h_{0}(p) \mid>M_{0}\right\}$, and hence $e^{i \boldsymbol{A} \alpha}$ leaves $D\left(h_{0}\right)$ invariant. Conditions (c) and (d) are satisfied because of the regularity assumptions (2) and (3) on $V$. (These hypotheses can be easily verified for a class of long range potentials with sufficient regularity at infinity.) 
Let us verify property (e). By hypothesis there exist $\alpha>0, \delta_{0}>0$ such that

$$
P_{h_{0}}\left(E, \delta_{0}\right) i\left[h_{0}, A\right]^{0} P_{h_{0}}\left(E, \delta_{0}\right) \geqq \alpha P_{h_{0}}\left(E, \delta_{0}\right) .
$$

For any smooth function $\tilde{P}$ such that $\tilde{P}=1$ on $(E-\delta, E+\delta) \delta<\delta_{0}$ and $\tilde{P}=0$ on $\mathbf{R} /\left(E-\delta_{0}, E+\delta_{0}\right)$, we have:

$$
\tilde{P}_{h_{0}} i\left[h_{0}, A\right]^{0} \tilde{P}_{h_{0}} \geqq \alpha \tilde{P}_{h_{0}}^{2} \text { and } P(E, \delta)=P(E, \delta) \tilde{P} .
$$

Note that $\tilde{P}_{H}-\tilde{P}_{h_{0}}$ is a compact operator since $V$ is $h_{0}$ compact and $\tilde{P}(\lambda)$ is a smooth function of compact support.

Then:

$$
\begin{aligned}
& P_{H}(E, \delta) i\left[h_{0}, A\right]^{0} P_{H}(E, \delta) \\
& \quad=P_{H}(E, \delta) \tilde{P}_{H} \sum_{i} g_{i}^{2}(p) \tilde{P}_{H} P_{H}(E, \delta) \\
& \quad=P_{H}(E, \delta) \tilde{P}_{h_{0}} \sum_{i} g_{i}^{2}(p) \tilde{P}_{h_{0}} P_{H}(E, \delta)+P_{H}(E, \delta) K^{\prime} P_{H}(E, \delta) \\
& \quad \geqq \alpha P_{H}(E, \delta) \tilde{P}_{h_{0}}^{2} P_{H}(E, \delta)+P_{H}(E, \delta) K^{\prime} P_{H}(E, \delta) \\
& \quad \geqq \alpha P_{H}^{2}(E, \delta)+P_{H}(E, \delta) K^{\prime \prime} P_{H}(E, \delta) .
\end{aligned}
$$

By hypothesis (2) $[V, A]$ is $h_{0}$ compact, hence there exist numbers $\alpha, \delta>0$ and a compact operator $K$ so that condition (e) holds. This proves Theorem I.2. The Corollary I.3 follows from Theorem I.2 and the abstract theorem since $D(A)$ contains $D(|x|)$, and hence $A(1+|x|)^{-1}$ is a bounded operator.

\section{Three-Body Schrödinger Operators}

Let $x_{i}, m_{i}$ be the coordinates and mass of the $i$-th particle where $x_{i} \in \mathbf{R}^{n}, i \in\{1,2,3\}$. For each pair of particles $(i, j)=\alpha$ (such pairs are always denoted by Greek letters), we will denote

$$
\begin{gathered}
x_{\alpha}=x_{i}-x_{j} ; \quad y_{\alpha}=x_{k}-\frac{m_{i} x_{i}+m_{j} x_{j}}{m_{i}+m_{j}} \quad k \notin \alpha \\
m_{\alpha}^{-1}=m_{i}^{-1}+m_{j}^{-1} \\
n_{\alpha}^{-1}=m_{k}^{-1}+\left(m_{i}+m_{j}\right)^{-1}
\end{gathered}
$$

when one removes the center of mass of the system, the Hilbert space is then

$$
\mathscr{H}=L^{2}\left(\mathbf{R}^{2 n}, d^{n} x_{\alpha} d^{n} y_{\alpha}\right) \quad \forall \alpha .
$$

$k_{\alpha}$ and $p_{\alpha}$ will denote $-i \nabla_{x_{\alpha}}$ and $-i \nabla_{y_{\alpha}}$.

In $\mathscr{H}$, the Hamiltonian of the system is written

$$
\begin{gathered}
H=H_{0}+V \\
H_{0}=\frac{1}{2 m_{\alpha}} k_{\alpha}^{2}+\frac{1}{2 n_{\alpha}} p_{\alpha}^{2} \quad \forall \alpha .
\end{gathered}
$$

The dilation group acts in the same way independently of the representation $L^{2}\left(d^{n} x_{\alpha}, d^{n} y_{\alpha}\right)$ of $\mathscr{H}$. Let $A$ be its generator normalized so that $i\left[H_{0}, A\right]=H_{0}$. We 
have $A=A_{\alpha}^{1}+A_{\alpha}^{2}$ where $A_{\alpha}^{1}$ and $A_{\alpha}^{2}$ are the generators of the dilation group on $L^{2}\left(d^{n} x_{\alpha}\right)$ and $L^{2}\left(d^{n} y_{\alpha}\right)$, respectively.

Hypotheses on the potential $V$

Suppose that $V=\sum_{\alpha} v_{\alpha}$ where, for each $\alpha, v_{\alpha}$ is an operator acting on $L^{2}\left(d^{n} x_{\alpha}\right)$ and satisfying hypotheses (i)-(iii) of Example 2.

We will further denote:

$$
H_{\alpha}=H_{0}+v_{\alpha}=h_{\alpha}+\frac{p_{\alpha}^{2}}{2 n_{\alpha}} ; \quad h_{\alpha}=\frac{k_{\alpha}^{2}}{2 m_{\alpha}}+v_{\alpha} .
$$

By Theorem I.1, the eigenvalues of $h_{\alpha}$ have finite multiplicity and can only accumulate at 0 .

Theorem I.3. Let $H=H_{0}+V$ on $L^{2}\left(d^{n} x_{\alpha}, d^{n} y_{\alpha}\right)$ where $V$ is a symmetric operator satisfying the above hypotheses. Then $A=A_{\alpha}^{1}+A_{\alpha}^{2}$ is a conjugate operator for $H$ at all $E \in \mathbf{R}$ with

$$
E \notin \bigcup_{\alpha} \sigma_{p}\left(h_{\alpha}\right) \cup\{0\}
$$

Corollary I.4. 1. The point spectrum of $H=H_{0}+\sum_{\alpha} v_{\alpha}$ can accumulate only at 0 or at eigenvalues of subsystems.

2. For all intervals $[a, b] \subset \mathbf{R} \backslash\left\{\sigma_{p}(H) \bigcup_{\alpha} \sigma_{p}\left(h_{\alpha}\right) \cup\{0\}\right\}$, there is a $c_{0}$ so that

$$
\sup _{\substack{\operatorname{Re} z \in[a, b] \\ \operatorname{Im} z \neq 0}}\left\|(1+|x|)^{-1}(H-z)^{-1}(1+|x|)^{-1}\right\| \leqq c_{0} .
$$

Under the hypotheses made on the two-body potential $v_{\alpha}$, conditions (a)-(d) are satisfied in the same way that they were in the two-body problem. Let us now prove that condition (e) holds.

Proposition 4.1. Let $E \in \mathbf{R}$, and let $c_{\alpha}$ be an $h_{\alpha}$-compact operator in $L^{2}\left(\mathbf{R}^{n}, d^{n} x_{\alpha}\right)$. Then for every $\varepsilon>0$ there is $\delta_{0}>0$, a finite rank spectral projection $e_{\alpha}^{N_{0}}$ for $h_{\alpha}$ and an operator $K$ compact in $\mathscr{H}=L^{2}\left(\mathbf{R}^{2 n}, d^{n} x_{\alpha} d^{n} y_{\alpha}\right)$ so that

$$
P_{H} c_{\alpha} P_{H}=P_{H} E_{\alpha}^{N} c_{\alpha} E_{\alpha}^{N} P_{H}+P_{H} K P_{H}+o(\varepsilon)
$$

where: $e_{\alpha}^{N_{0}}$,

(i) $E_{\alpha}^{N}=e_{\alpha}^{N} \otimes \mathbb{1} y_{\alpha}$ where $e_{\alpha}^{N}$ is a finite rank spectral projection for $h_{\alpha}$ that contains

(ii) $P_{H}$ is any spectral projection for $H$ onto any Borel set contained in $\left(E-\delta_{0}, E+\delta_{0}\right)$;

(iii) $\|o(\varepsilon)\| \leqq \frac{\varepsilon}{6}$.

Proof. Since $c_{\alpha}$ is an $h_{\alpha}$-compact operator, we can find $e_{\alpha}^{N_{0}}$ so that

$$
\left\|e_{\alpha}^{N_{0}} c_{\alpha} e_{\alpha}^{N_{0}}-P_{h_{\alpha}}^{p} c_{\alpha} P_{h_{\alpha}}^{p}\right\| \leqq \frac{\varepsilon}{12} .
$$


Furthermore, from general properties of the continuous spectrum, one can find a $\delta_{0}>0$ and a smooth function $\tilde{P}$ with $\tilde{P}=1$ on $\left(E-\delta_{0}, E+\delta_{0}\right)$ and 0 on $\mathbf{R} \backslash\left(E-2 \delta_{0}, E+2 \delta_{0}\right)$ so that

$$
\left\|\tilde{P}_{H_{\alpha}}\left\{c_{\alpha}-P_{h_{\alpha}}^{p} c_{\alpha} P_{h_{\alpha}}^{p}\right\} \tilde{P}_{H_{\alpha}}\right\| \leqq \frac{\varepsilon}{12} .
$$

Hence for all $\delta \leqq \delta_{0}$ and all spectral projections $P_{H}$ on $(E-\delta, E+\delta)$ we have

$$
P_{H} c_{\alpha} P_{H}=P_{H} E_{\alpha}^{N} c_{\alpha} E_{\alpha}^{N} P_{H}+P_{H}\left\{c_{\alpha}-P_{h_{\alpha}}^{p} c_{\alpha} P_{h_{\alpha}}^{p}\right\} P_{H}+o_{1}(\varepsilon)
$$

with $\left\|o_{1}(\varepsilon)\right\| \leqq \frac{\varepsilon}{12}$.

On the other hand $P_{H}=P_{H} \tilde{P}_{H}$ and thus

$$
\begin{aligned}
P_{H}\left\{c_{\alpha}-P_{h_{\alpha}}^{p} c_{\alpha} P_{h_{\alpha}}^{p}\right\} P_{H}= & P_{H}\left(\tilde{P}_{H}-\tilde{P}_{H_{\alpha}}\right)\left\{c_{\alpha}-P_{h_{\alpha}}^{p} c_{\alpha} P_{h_{\alpha}}^{p}\right\} P_{H} \\
& +P_{H} \tilde{P}_{\alpha_{\alpha}}\left\{c_{\alpha}-P_{h_{\alpha}}^{p} c_{\alpha} P_{h_{\alpha}}^{p}\right\}\left(\tilde{P}_{H}-\tilde{P}_{H_{\alpha}}\right) P_{H} \\
& +P_{H} \tilde{P}_{H_{\alpha}}\left\{c_{\alpha}-P_{h_{\alpha}}^{p} c_{\alpha} P_{h_{\alpha}}^{p}\right\} \tilde{P}_{H_{\alpha}} P_{H},
\end{aligned}
$$

where the first two terms on the right hand side are compact operators in $\mathscr{H}$ and the last has norm less than $\frac{\varepsilon}{12}$.

Proposition 4.2. For all $\varepsilon>0$, we can find $\delta_{0}>0, E_{\alpha}^{N_{0}}=e_{\alpha}^{N_{0}} \otimes \mathbb{1}_{y_{\alpha}}$, and a compact operator $K$ so that:

$$
\begin{aligned}
P_{H} i\left[H_{0}+\sum_{\alpha} v_{\alpha}, A\right] P_{H}= & P_{H}\left(1-\sum_{\alpha} E_{\alpha}^{N_{0}}\right) H_{0}\left(1-\sum_{\alpha} E_{\alpha}^{N_{0}}\right) P_{H} \\
& +\sum_{\alpha} P_{H} E_{\alpha}^{N_{0}}\left\{H_{0}+i\left[v_{\alpha}, A_{\alpha}^{1}\right]\right\} E_{\alpha}^{N_{0}} P_{H} \\
& +o(\varepsilon)+P_{H} K P_{H}
\end{aligned}
$$

with $\|o(\varepsilon)\|<\varepsilon$, for any spectral projection $P_{H}$ onto an interval contained in $\left(E-\delta_{0}, E+\delta_{0}\right)$.

Proof. We have

$$
\begin{aligned}
H_{0}= & \left(1-\sum_{\alpha} E_{\alpha}^{N}\right) H_{0}\left(1-\sum_{\alpha} E_{\alpha}^{N}\right)+\sum_{\alpha} E_{\alpha}^{N} H_{0} E_{\alpha}^{N} \\
& +\sum_{\alpha}\left\{E_{\alpha}^{N} H_{0}\left(1-E_{\alpha}^{N}\right)+\left(1-E_{\alpha}^{N}\right) H_{0} E_{\alpha}^{N}\right\} \\
& -\sum_{\alpha \neq \beta} \sum_{\alpha} E_{\alpha}^{N} H_{0} E_{\beta}^{N} .
\end{aligned}
$$

The terms in the last sum are all compact operators in $\mathscr{H}$ and $E_{\alpha}^{N} H_{0}\left(1-E_{\alpha}^{N}\right)$ $=-E_{\alpha}^{N} v_{\alpha}\left(1-E_{\alpha}^{N}\right)$ since $E_{\alpha}^{N}$ commutes with $H_{\alpha}=H_{0}+v_{\alpha}$. We consider spectral projections $e_{\alpha}^{N}$ for $h_{\alpha}$ so that

$$
\sum_{\beta} E_{\beta}^{N} H_{0}\left(1-E_{\beta}^{N}\right)=\sum_{\beta} P_{h_{\beta}}^{p}\left(-v_{\beta}\right) P_{h_{\beta}}^{c}+o(\varepsilon)
$$

with $\|o(\varepsilon)\|<\frac{\varepsilon}{2}$. 
Next, we apply Proposition 4.1 to each of the operators

$$
c_{\alpha}=i\left[v_{\alpha}, A_{\alpha}^{1}\right]-P_{h_{\alpha}}^{p} v_{\alpha} P_{h_{\alpha}}^{c}-P_{h_{\alpha}}^{c} v_{\alpha} P_{h_{\alpha}}^{p} .
$$

By Proposition 4.1, we can find $E_{\alpha}^{N_{0}}$ and $\delta_{0}>0$ satisfying Proposition 4.2.

Proposition 4.3. Let $\alpha_{0}=\operatorname{dist}\left(E,\{0\} \bigcup_{\alpha} \sigma_{p}\left(h_{\alpha}\right)\right)$. We can find $\delta_{0}$ so that

$$
\sum_{\alpha} P_{H} E_{\alpha}^{N}\left\{H_{0}+i\left[v_{\alpha}, A_{\alpha}^{1}\right]\right\} E_{\alpha}^{N} P_{H} \geqq \sum_{\alpha} \frac{\alpha_{0}}{2} P_{H} E_{\alpha}^{N} P_{H}+P_{H} K P_{H} ; P_{H}=P_{H}\left(E, \delta_{0}\right)
$$

Proof. If we choose $\delta_{0}$ so that

$$
\begin{aligned}
& \delta_{0} \leqq \frac{1}{4} \inf _{\alpha} \inf _{i \neq j}\left|\lambda_{\alpha}^{i}-\lambda_{\alpha}^{j}\right| \\
& \delta_{0} \leqq \frac{\alpha_{0}}{4} .
\end{aligned}
$$

$\lambda_{\alpha}^{i}$, being the eigenvalues of $h_{\alpha} e_{\alpha}^{N}$.

If we pick a function $\tilde{P}$ equal to 1 on $\left(E-\delta_{0}, E+\delta_{0}\right)$ and 0 on $\mathbf{R} \backslash\left(E-2 \delta_{0}, E+2 \delta_{0}\right)$,

$$
\tilde{P}_{H_{\alpha}} E_{\alpha}^{i}\left\{H_{0}+i\left[v_{\alpha}, A_{\alpha}^{1}\right]\right\} E_{\alpha}^{j} \tilde{P}_{H_{\alpha}}=0 \quad \text { if } \quad i \neq j
$$

since $E_{\alpha}^{j} \tilde{P}_{H_{\alpha}}$ and $E_{\alpha}^{i} \tilde{P}_{H_{\alpha}}$ viewed as functions of $p_{\alpha}^{2}$ have support in disjoint intervals $\left(E_{\alpha}^{i} \tilde{P}\left(H_{\alpha}\right)=\tilde{P}\left(\lambda_{\alpha}^{i}+\frac{p_{\alpha}^{2}}{2 n_{\alpha}}\right) E_{\alpha}^{i}\right)$. Furthermore, by the Virial Theorem,

$$
\begin{gathered}
\tilde{P}_{H_{\alpha}} E_{\alpha}^{N}\left\{H_{0}+i\left[v_{\alpha}, A_{\alpha}^{1}\right]\right\} E_{\alpha}^{N} \tilde{P}_{H_{\alpha}} \\
=\sum_{i} \tilde{P}_{H_{\alpha}} E_{\alpha}^{i} i\left[h_{\alpha}, A_{\alpha}^{1}\right] E_{\alpha}^{i} \tilde{P}_{H_{\alpha}} \\
\quad+\sum_{i} \tilde{P}_{H_{\alpha}} E_{\alpha}^{i} \frac{p_{\alpha}^{2}}{2 n_{\alpha}} E_{\alpha}^{i} \tilde{P}_{H_{\alpha}} \\
=\sum_{i} \tilde{P}_{H_{\alpha}} E_{\alpha}^{i} \frac{p_{\alpha}^{2}}{2 n_{\alpha}} E_{\alpha}^{i} \tilde{P}_{H_{\alpha}} \\
\geqq \frac{\alpha_{0}}{2} \tilde{P}_{H_{\alpha}} E_{\alpha}^{N} \tilde{P}_{H_{\alpha}} .
\end{gathered}
$$

Propositions 4.2 and 4.3 enable us to find, for all $\varepsilon>0,\left(e_{\alpha}^{N}\right)$ and $\delta_{0}>0$ so that $P_{H}(E, \delta) i[H, A]^{0} P_{H}(E, \delta)$

$$
\begin{aligned}
\geqq & P_{H}\left(1-\sum_{\alpha} E_{\alpha}^{N}\right) H_{0}\left(1-\sum_{\alpha} E_{\alpha}^{N}\right) P_{H} \\
& +\frac{\alpha_{0}}{2} \sum_{\alpha} P_{H} E_{\alpha}^{N} P_{H} \\
& +P_{H} K P_{H}+P_{H} O(\varepsilon) P_{H},
\end{aligned}
$$

where $\|o(\varepsilon)\|<\varepsilon$, for all $\delta<\delta_{0}$. 
To verify condition (e), since $\varepsilon>0$ is arbitrary, it now suffices to show that there is a finite constant $c_{0}$ so that

$$
P_{H} \leqq c_{0}\left\{P_{H}\left(1-\sum_{\alpha} E_{\alpha}^{N}\right) H_{0}\left(1-\sum_{\alpha} E_{\alpha}^{N}\right) P_{H}+\sum_{\alpha} P_{H} E_{\alpha}^{N} P_{H}\right\}
$$

which is immediate if $E \neq 0$; the constant $c_{0}$ evidently does not depend on $N$ and $\delta$.

\section{Proof of Theorem I}

We start the proof of the abstract theorem by the following proposition which is useful in applications to verify the hypothesis (c) when $D(A) \cap D(H)$ is not explicitly known.

Proposition II.1. Let $H$ and $A$ be self-adjoint operators that satisfy conditions (a), (b) and the following conditions $\left(\mathrm{c}^{\prime}\right)$.

(c') There is a set $\mathscr{S} \subset D(A) \cap D(H)$ such that

i) $e^{+i A \alpha} \mathscr{S} \subset \mathscr{S}$

ii) $\mathscr{S}$ is a core for $H$,

iii) the form $i[H, A]$ on $\mathscr{S}$ is bounded below and closeable, and the associated self-adjoint operator $i[H, A]_{\mathscr{S}}^{0}$ satisfies

$$
D\left(i[H, A]_{\mathscr{S}}^{0}\right) \supset D(H)
$$

then for all $\Phi, \Psi \in D(A) \cap D(H)$

$$
(\Phi \mid i[H, A] \Psi)=\left(\Phi \mid i[H, A]_{\mathscr{S}}^{0} \Psi\right)
$$

and hence the form $i[H, A]$ on $D(A) \cap D(H)$ is closeable and the associated selfadjoint operator satisfies:

$$
i[H, A]^{0}=i[H, A]_{\mathscr{S}}^{0} .
$$

Proof. It suffices to check that for each $\Phi, \Psi \in D(A) \cap D(H)$

$$
(\Phi \mid i[H, A] \Psi)=\left(\Phi \mid i[H, A]_{\mathscr{S}}^{0} \Psi\right) .
$$

By hypothesis (b), the operators $H e^{+i A \alpha}(H+i)^{-1}$ are closed and everywhere defined, hence bounded by the closed graph theorem. For each $\Psi \in \mathscr{H}$, by (b)

sup $\left\|H e^{+i A \alpha}(H+i)^{-1} \Psi\right\|<\infty$ and by the principle of uniform boundedness in $\alpha \in[-1,+1]$

Banach spaces, this family of operators is uniformly bounded: there is a $c_{0}<\infty$ such that:

$$
\sup _{\alpha \in[-1,+1]}\left\|H e^{+i A \alpha}(H+i)^{-1}\right\| \leqq c_{0} .
$$

Consequently, for each $\Phi, \Psi \in D(A) \cap D(H),\left(H(\alpha)=e^{-i A \alpha} H e^{+i A \alpha}\right)$,

$$
\begin{aligned}
\lim _{\alpha \rightarrow 0} & \frac{1}{\alpha}(\Phi \mid(H(\alpha)-H) \Psi) \\
& =\lim _{\alpha \rightarrow 0} \frac{1}{\alpha}\left(\Phi \mid\left(e^{-i A \alpha}-1\right) H e^{+i A \alpha} \Psi\right)+\frac{1}{\alpha}\left(\Phi \mid H\left(e^{+i A \alpha}-1\right) \Psi\right) \\
& =(\Phi \mid i[H, A] \Psi) .
\end{aligned}
$$


Since $H e^{+i A \alpha} \Psi$ is uniformly bounded in $\alpha$, this family of vectors converges weakly to $H \Psi$ when $\alpha \rightarrow 0$.

For each $\Phi, \Psi \in D(H)$ there are sequences $u_{n}$ and $v_{n}$ such that

$$
\left\|(H+i)\left(u_{n}-\Phi\right)\right\| \rightarrow 0, \quad\left\|(H+i)\left(v_{n}-\Psi\right)\right\| \rightarrow 0
$$

with $u_{n}, v_{n} \in \mathscr{S}$. Thus :

$$
\frac{1}{\alpha}(\Phi \mid(H(\alpha)-H) \Psi)=\lim _{n \rightarrow \infty} \frac{1}{\alpha}\left(u_{n} \mid(H(\alpha)-H) v_{n}\right) .
$$

By hypothesis $\left(\mathrm{c}^{\prime}\right)$, the derivative

$$
\frac{d}{d \alpha}\left(u_{n} \mid H(\alpha) v_{n}\right)=\left(u_{n} \mid e^{-i A \alpha} i[H, A]_{\mathscr{S}}^{0} e^{+i A \alpha} v_{n}\right)
$$

is a continuous function: one can then use the mean value theorem to obtain:

$$
\frac{1}{\alpha}(\Phi \mid(H(\alpha)-H) \Psi)=\lim _{n \rightarrow \infty}\left(u_{n} \mid e^{-i A \alpha_{n}} i[H, A]_{\mathscr{S}}^{0} e^{+i A \alpha_{n}} v_{n}\right)
$$

where $\alpha_{n} \in[0, \alpha]$. Since $D\left(i[H, A]_{\mathscr{S}}^{0}\right) \supset D(H)$, (II.1) assures that as $n \rightarrow \infty, \alpha \rightarrow 0$

$$
\begin{aligned}
(\Phi \mid i[H, A] \Psi) & =\lim _{\alpha \rightarrow 0} \frac{1}{\alpha}(\Phi \mid(H(\alpha)-H) \Psi) \\
& =\left(\Phi \mid i[H, A]_{\mathscr{S}}^{0} \Psi\right) .
\end{aligned}
$$

Proposition II.2. Suppose that the two self-adjoint operators $H$ and $A$ satisfy conditions (a)-(c). Then $(H-z)^{-1}$ leaves $D(A)$ invariant for all $z \notin \sigma(H)$.

Proof. Since $A$ is self-adjoint, it suffices to show that the family of operators

$$
e^{-i A \alpha}(H-z)^{-1}(A+i)^{-1}=(H(\alpha)-z)^{-1} e^{-i A \alpha}(A+i)^{-1}
$$

is strongly differentiable; it suffices to show that the family $H(\alpha)(H-z)^{-1}$ is strongly differentiable, or equivalently to show that for each $\Psi \in D(H)$

$$
\lim _{\alpha \rightarrow 0}\left\|\frac{H(\alpha)-H}{\alpha} \Psi-i[H, A]^{0} \Psi\right\|=0 .
$$

Let $\Psi_{n} \in D(A) \cap D(H)$ so that $\left\|(H+i)\left(\Psi_{n}-\Psi\right)\right\| \rightarrow 0$. Then

$$
\frac{H(\alpha)-H}{\alpha} \Psi-i[H, A]^{0} \Psi=\lim _{n \rightarrow \infty} \frac{H(\alpha)-H}{\alpha} \Psi_{n}-i[H, A]^{0} \Psi_{n}
$$

exactly as in Proposition II.1. Since $e^{+i A \alpha}$ leaves $D(A) \cap D(H)$ invariant for each $\Phi \in D(A) \cap D(H),\|\Phi\|=1$, there exist $\alpha_{n, \Phi} \in[0, \alpha]$ so that

$$
\left(\Phi \mid \frac{H(\alpha)-H}{\alpha} \Psi_{n}\right)=\left(\Phi \mid e^{-i A \alpha_{n}} \varphi i[H, A]^{0} e^{+i A \alpha_{n, \Phi}} \Psi_{n}\right) .
$$

Bound (II.1) and the hypothesis that $D(H) \subset D\left(i[H, A]^{0}\right)$, together imply

$$
\|(H(\alpha)-H) \Psi\| \leqq \alpha c_{0}\|(H+i) \Psi\|
$$


for all $\Psi \in D(H)$. Furthermore,

$$
\begin{aligned}
& \|\left(\Phi \mid \frac{H(\alpha)-H}{\alpha} \Psi_{n}\right)-\left(\Phi \mid i[H, A]^{0} \Psi_{n}\right) \mid \\
& \quad \leqq c\left\|(H+i)\left(\Psi_{n}-\Psi\right)\right\|+\left\|\left(\Phi \mid\left\{e^{-i A \alpha_{n, \Phi}} i[H, A]^{0} e^{+i A \alpha_{n, \Phi}}-i[H, A]^{0}\right\} \Psi\right)\right\| \\
& \quad \leqq o\left(\frac{1}{n}\right)+\sup _{\alpha^{\prime} \in[0, \alpha]}\left\|\left\{e^{-i A \alpha^{\prime}} i[H, A]^{0} e^{+i A \alpha^{\prime}}-i[H, A]^{0}\right\} \Psi\right\| \\
& \quad \leqq o\left(\frac{1}{n}\right)+\sup _{\alpha^{\prime} \in[0, \alpha]}\left\|i[H, A]^{0}\left(e^{+i A \alpha^{\prime}}-\mathbb{1}\right) \Psi\right\|+\left\|\left(e^{-i A \alpha^{\prime}}-\mathbb{1}\right) i[H, A]^{0} \Psi\right\| \\
& \quad \leqq o\left(\frac{1}{n}\right)+o(\alpha)+\sup _{\alpha^{\prime} \in[0, \alpha]} c_{0}\left\|H\left(e^{+i A \alpha^{\prime}}-1\right) \Psi\right\| .
\end{aligned}
$$

But finally

$$
\begin{aligned}
\left\|H\left(e^{+i A \alpha^{\prime}}-1\right) \Psi\right\| & =\left\|\left(H\left(\alpha^{\prime}\right)-e^{-i A \alpha^{\prime}} H\right) \Psi\right\| \\
& \leqq\left\|\left(H\left(\alpha^{\prime}\right)-H\right) \Psi\right\|+\left\|\left(1-e^{-i A \alpha^{\prime}}\right) H \Psi\right\|
\end{aligned}
$$

which goes to zero as $\alpha \rightarrow 0$ by (II.2).

Proposition II.3. If the operators $H, A$ satisfy conditions (a)-(c), then $(A \pm i \lambda)^{-1}$ leaves $D(H)$ invariant for sufficiently large $\lambda$. Further $(H+i) i \lambda(A+i \lambda)^{-1}(H+i)^{-1}$ converges strongly to 1 as $|\lambda| \rightarrow \infty$.

Proof. By Proposition II.2, we have in the operator sense

$$
\begin{aligned}
(A & +i \lambda)^{-1}(H+i)^{-1}-(H+i)^{-1}(A+i \lambda)^{-1} \\
& =(A+i \lambda)^{-1}\left\{(H+i)^{-1} A-A(H+i)^{-1}\right\}(A+i \lambda)^{-1} \\
& =(A+i \lambda)^{-1}(H+i)^{-1}[A, H](H+i)^{-1}(A+i \lambda)^{-1},
\end{aligned}
$$

where the last equality holds in the sense of quadratic form on $\mathscr{H}$. By condition (c), there is a bounded operator $B(\lambda)=[A, H]^{0}(H+i)^{-1}(A+i \lambda)^{-1}$ with $\|B(\lambda)\| \rightarrow 0$ as $|\lambda| \rightarrow \infty$ such that

$$
(A+i \lambda)^{-1}(H+i)^{-1}(1-B(\lambda))=(H+i)^{-1}(A+i \lambda)^{-1} .
$$

This proves Proposition II.3 since when $|\lambda|$ is sufficiently large, $1-B(\lambda)$ is invertible and $i \lambda(A+i \lambda)^{-1}(1-B(\lambda))^{-1}$ converges strongly to $\mathbb{1}$ as $|\lambda| \rightarrow \infty$.

Proposition II.4 (The Virial Theorem). Let $H$ and $A$ be two self-adjoint operators satisfying conditions (a)-(c). Then

1. For all $\Psi \in D(H)$

$$
[H, A]^{0} \Psi=\lim _{|\lambda| \rightarrow \infty}\left[H, \operatorname{Ai\lambda }(A+i \lambda)^{-1}\right] \Psi .
$$

2. If $\Psi$ is an eigenvector of $H$, we have

$$
\left(\Psi \mid[H, A]^{0} \Psi\right)=0 .
$$


Proof. Let $\Psi \in D(H), \Phi \in D(A) \cap D(H)$. By Propositions II.2 and II.3, for sufficiently large $|\lambda|$,

$$
\begin{aligned}
&\left(\Phi \mid\left[H, A i \lambda(A+i \lambda)^{-1}\right] \Psi\right) \\
&=\left(\Phi \mid\left\{H A i \lambda(A+i \lambda)^{-1}-A i \lambda(A+i \lambda)^{-1} H\right\} \Psi\right) \\
&=\left(\Phi \mid(H A-A H) i \lambda(A+i \lambda)^{-1} \Psi\right) \\
&+\left(A \Phi \mid\left\{H i \lambda(A+i \lambda)^{-1}-i \lambda(A+i \lambda)^{-1} H\right\} \Psi\right) \\
&=\left(\Phi \mid[H, A]^{0} i \lambda(A+i \lambda)^{-1} \Psi\right) \\
&+\left(\Phi \mid A(A+i \lambda)^{-1}[H, A]^{0} i \lambda(A+i \lambda)^{-1} \Psi\right) .
\end{aligned}
$$

Since $[A, H]^{0} i \lambda(A+i \lambda)^{-1} \Psi \rightarrow[A, H]^{0} \Psi$ by Proposition II.3 and condition (c), and since $A(A+i \lambda)^{-1} \stackrel{s}{\longrightarrow} 0$, Proposition II.3 implies that

$$
\lim _{|\lambda| \rightarrow \infty}\left[H, A i \lambda(A+i \lambda)^{-1}\right] \Psi=[H, A]^{0} \Psi .
$$

Proving (1). Finally, if $\Psi$ is an eigenvector for $H, \Psi \in D(H)$ and $H \Psi=E \Psi$, so that

$$
\left(\Psi \mid[H, A]^{0} \Psi\right)=\lim _{|\lambda| \rightarrow \infty}\left(\Psi \mid\left[H, A i \lambda(A+i \lambda)^{-1}\right] \Psi\right)=0
$$

\section{Proof of Part (1) of Theorem 1}

If one supposes that the self-adjoint operators $H, A$ satisfy conditions (a)-(c), and if furthermore they satisfy condition(e) at $E \in \mathbf{R}$ then the point spectrum in $(E-\delta, E+\delta)$ is finite. Suppose not. Then there is a sequence $\Psi_{n}$ of orthonormal eigenvectors $H \Psi_{n}=E_{n} \Psi_{n}$. By Proposition II.4

$$
\begin{aligned}
0 & =\left(\Psi_{n} \mid i[H, A]^{0} \Psi_{n}\right)=\left(\Psi_{n} \mid P_{H}(E, \delta) i[H, A]^{0} P_{H}(E, \delta) \Psi_{n}\right) \\
& \geqq \alpha\left\|\Psi_{n}\right\|^{2}+\left(\Psi_{n} \mid K \Psi_{n}\right) .
\end{aligned}
$$

Since the $\Psi_{n}$ are orthonormal, $\Psi_{n} \stackrel{w}{\longrightarrow} 0$ in $\mathscr{H}$ and since $K$ is compact $\lim _{n \rightarrow \infty}\left(\Psi_{n} \mid i[H, A]^{0} \Psi_{n}\right) \geqq \alpha$ which is impossible.

Proposition II.5 (Quadratic Estimate). Let $\mathrm{H}$ be a self-adjoint operator with domain $D(H)$ and $B^{*} B$ a bounded positive operator on $\mathscr{H}$. Then

1. $H-z-i \varepsilon B^{*} B$ is invertible if $\operatorname{Im} z$ and $\varepsilon$ have the same sign.

2. If $\operatorname{Im} z$ and $\varepsilon$ have the same sign, let

$$
G_{z}(\varepsilon)=\left(H-z-i \varepsilon B^{*} B\right)^{-1} .
$$

Let $B^{\prime}$ an operator with $B^{\prime *} B^{\prime} \leqq B^{*} B$ and $C$ any bounded self-adjoint operator on $\mathscr{H}$, then:

$$
\left\|B^{\prime} G_{z}(\varepsilon) C\right\| \leqq \frac{1}{\sqrt{\varepsilon}}\left\|C G_{z}(\varepsilon) C\right\|^{1 / 2}
$$


Proof. Since $B^{*} B$ is bounded $H-z-i \varepsilon B^{*} B$ is a closed operator on $D(H)$. When $\Psi \in D(H)$ and $\varepsilon$ and $\operatorname{Im} z$ have the same sign, we have

$$
\begin{aligned}
\left\|\left(H-z-i \varepsilon B^{*} B\right) \Psi\right\|^{2}= & \|(H-\operatorname{Re} z) \Psi\|^{2}+\left\|\left(\operatorname{Im} z+\varepsilon B^{*} B\right) \Psi\right\|^{2} \\
& -2 \operatorname{Im}\left((H-\operatorname{Re} z) \Psi \mid \varepsilon B^{*} B \Psi\right) \\
\geqq & (\operatorname{Im} z)^{2}\|\Psi\|^{2} .
\end{aligned}
$$

From this inequality and the fact that $H-z-i \varepsilon B^{*} B$ is a closed operator, it follows that $H-z-i \varepsilon B^{*} B$ is injective with closed range in $\mathscr{H}$. By the open mapping theorem, its inverse exists as a bounded operator from $\operatorname{Rang}\left(H-z-i \varepsilon B^{*} B\right)$ into $\mathscr{H}_{+2}$. But $\operatorname{Rang}\left(H-z-i \varepsilon B^{*} B\right)=\mathscr{H}$ since if $\Phi_{0} \in \mathscr{H}$ is orthogonal to this range, then $\Phi_{0} \in D(H)$ and $\left(H-\bar{z}+i \varepsilon B^{*} B\right) \Phi_{0}=0$ which by (II.4) implies $\Phi_{0}=0$. Finally:

$$
\begin{aligned}
\left\|B^{\prime} G_{z}(\varepsilon) C\right\|^{2} & =\left\|C G_{z}^{*}(\varepsilon) B^{\prime *} B^{\prime} G_{z}(\varepsilon) C\right\| \\
& \leqq \frac{1}{\varepsilon}\left\|C\left(H-\bar{z}+i \varepsilon B^{*} B\right)^{-1}\left(\operatorname{Im} z+\varepsilon B^{*} B\right)\left(H-z-i \varepsilon B^{*} B\right)^{-1} C\right\| \\
& \leqq \frac{1}{2 \varepsilon}\left\|C\left(G_{z}^{*}(\varepsilon)-G_{z}(\varepsilon)\right) C\right\| \\
& \leqq \frac{1}{\varepsilon}\left\|C G_{z}(\varepsilon) C\right\|=\frac{1}{\varepsilon}\left\|C G_{z}^{*}(\varepsilon) C\right\|
\end{aligned}
$$

Proof of Part (2) of Theorem 1

We will prove the following lemma which clearly implies statement (2) of Theorem 1.

Lemma. Let $H$ be a self-adjoint operator with conjugate operator $A$ in a neighborhood of $E$, i.e. suppose $H, A$, and $E$ satisfy conditions (a)-(e). Then for any $E^{\prime} \in(E-\delta, E+\delta) \cap \sigma_{c}(H)$, there is a neighborhood $(a, b)$ of $E^{\prime}$ and a constant $c_{0}$ so that

$$
\sup _{\substack{\operatorname{Re} z \in[a, b] \\ \operatorname{Im} z \neq 0}}\left\||A+i|^{-1}(H-z)^{-1}|A+i|^{-1}\right\| \leqq c_{0} .
$$

Proof. By hypothesis (e), there are numbers $\alpha, \delta>0$ and a compact operator $K$ on $\mathscr{H}$ such that

$$
P_{H}(E, \delta) i[H, A]^{0} P_{H}(E, \delta) \geqq \alpha P_{H}^{2}(E, \delta)+P_{H}(E, \delta) K P_{H}(E, \delta),
$$

where $P_{H}(E, \delta)$ is the spectral projector of $H$ onto the interval $(E-\delta, E+\delta)$. By hypothesis $E^{\prime} \in \sigma_{c}(H)$, hence the spectral projector for $H$ onto $\left(E^{\prime}-\varepsilon, E^{\prime}+\varepsilon\right)$ converges weakly to zero as $\varepsilon \rightarrow 0$. Hence one can find $\delta^{\prime}>0$ and a smooth function $P \leqq 1, P=1$ on $\left(E^{\prime}-\delta^{\prime}, E^{\prime}+\delta^{\prime}\right), P=0$ on $\mathbf{R} /\left(E-\delta, E+\delta\right.$ ) so that (denoting by $P_{H}$ the operator associated to this $P$ )

$$
\pm P_{H} K P_{H} \leqq \frac{\alpha}{2} P_{H}^{2}
$$


and hence

$$
P_{H} i[H, A]^{0} P_{H} \geqq \frac{\alpha}{2} P_{H}^{2} .
$$

Let $B^{*} B=P_{H} i[H, A]^{0} P_{H}$.

By Proposition II.5, $G_{z}(\varepsilon)=\left(H-z-i \varepsilon B^{*} B\right)^{-1}$ exists if $\operatorname{Im} z$ and $\varepsilon$ have the same sign. Let

$$
F_{z}(\varepsilon)=|A+i|^{-1} G_{z}(\varepsilon)|A+i|^{-1}
$$

We have by Proposition II.5

$$
\left\|P_{H} G_{z}(\varepsilon)|A+i|^{-1}\right\| \leqq \frac{c}{\sqrt{\varepsilon}}\left\|F_{z}(\varepsilon)\right\|^{1 / 2} .
$$

Furthermore,

$$
\begin{aligned}
& \left\|\left(1-P_{H}\right) G_{z}(\varepsilon)|A+i|^{-1}\right\| \\
& \quad \leqq\left\|\left(1-P_{H}\right) G_{z}(0)\right\|\left\|\left(1-i \varepsilon B^{*} B G_{z}(\varepsilon)\right)|A+i|^{-1}\right\| \\
& \quad \leqq c\left\|\left(1-P_{H}\right) G_{z}(0)\right\| .
\end{aligned}
$$

Remark. (II.5) and (II.6) remain true if one replaces $P_{H}$ and $\left(1-P_{H}\right)$ by $(H+i) P_{H}$ and $(H+i)\left(1-P_{H}\right)$. If we restrict $\operatorname{Re} z$ to a closed interval $[a, b]$ strictly contained in $\left(E^{\prime}-\delta^{\prime}, E^{\prime}+\delta^{\prime}\right),\left(1-P_{H}\right) G_{z}(0)$ is uniformly bounded, and there is a constant $c$ so that:

$$
\left\|F_{z}(\varepsilon)\right\| \leqq \frac{c}{\varepsilon} \quad \operatorname{Re} z \in[a, b]
$$

Furthermore

$$
\frac{d}{d \varepsilon} F_{z}(\varepsilon)=|A+i|^{-1} G_{z}(\varepsilon) P_{H} i[H, A]^{0} P_{H} G_{z}(\varepsilon)|A+i|^{-1}
$$

We can write

$$
\begin{aligned}
P_{H}[H, A]^{0} P_{H}= & {[H, A]^{0}-\left(1-P_{H}\right)[H, A]^{0} P_{H} } \\
& -P_{H}[H, A]^{0}\left(1-P_{H}\right)-\left(1-P_{H}\right)[H, A]^{0}\left(1-P_{H}\right)
\end{aligned}
$$

so that by Eqs. (II.5) and (II.6) and the remarks following them, there are constants $c_{1}, c_{2}$ so that

$$
\begin{aligned}
\left\|\frac{d}{d \varepsilon} F_{z}(\varepsilon)\right\| \leqq & \left\||A+i|^{-1} G_{z}(\varepsilon) i[H, A]^{0} G_{z}(\varepsilon)|A+i|^{-1}\right\| \\
& +c_{1}+c_{2} \frac{1}{\sqrt{\varepsilon}}\left\|F_{z}(\varepsilon)\right\|^{1 / 2} .
\end{aligned}
$$

By condition (d) and Proposition II.6 (see the appendix), $G_{z}(\varepsilon): D(A) \rightarrow D(A) \cap D(H)$ and $\left[B^{*} B, A\right]$ is bounded as a map from $\mathscr{H}_{+2}$ into $\mathscr{H}_{-2}$. Hence in (II.8), we can write $[H, A]^{0}$ as $\left[H-z-i \varepsilon B^{*} B, A\right]+i \varepsilon\left[B^{*} B, A\right]$. Substituting this relation into 
(II.8), we find that

$$
\left\|\frac{d}{d \varepsilon} F_{z}(\varepsilon)\right\| \leqq \tilde{c}_{1}+\tilde{c}_{2} \frac{1}{\sqrt{\varepsilon}}\left\|F_{z}(\varepsilon)\right\|^{1 / 2}+\tilde{c}_{3}\left\|F_{z}(\varepsilon)\right\|
$$

for constants $\tilde{c}_{1}, \tilde{c}_{2}, \tilde{c}_{3}$ independent of $\varepsilon$ and $z$ such that $\operatorname{Re} z \in[a, b]$ and $\operatorname{Im} z$ and $\varepsilon$ with the same sign.

This differential inequality together with the relation (II.7) shows that there exists a constant $c_{0}$ so that

$$
\left\|F_{z}(\varepsilon)\right\| \leqq c_{0}
$$

for all $z$ with $\operatorname{Re} z \in[a, b], \operatorname{Im} z \neq 0$ and $\operatorname{Im} z, \varepsilon$ having the same sign.

\section{Appendix I}

Let $\left\{g_{i}(p)\right\} i \in\{1, \ldots, n\}$ be a $\mathscr{C}^{2}$ vector field, and let $\hat{A}$ be the symmetric operator defined on $L^{2}\left(\mathbf{R}^{n}, d^{n} p\right)$ by

$$
\begin{aligned}
\hat{A} & =\sum_{i=1}^{n} g_{i}(p) i \frac{\partial}{\partial p_{i}}+\frac{i}{2} \frac{\partial g_{i}}{\partial p_{i}}(p) \\
& =\frac{1}{2} \sum_{i}\left(g_{i} x_{i}+x_{i} g_{i}\right)
\end{aligned}
$$

If each $g_{i}$ is $\mathscr{C}^{2}$ the quadratic form defined by $\hat{A}$ admits a form domain containing the form domain of $x^{2}=\sum_{i=1}^{n} x_{i}^{2}$, the same holds for the quadratic form $\hat{A} x^{2}-x^{2} \hat{A}$. By the commutator theorem ([4, Vol. II] $), \hat{A}$ defines a self-adjoint operator $A$ which is essentially self-adjoint on any core for $x^{2}$. On the other hand, the system of differential equations

$$
\begin{aligned}
\frac{d}{d \alpha} \Gamma_{\alpha}^{i}(p) & =g_{i}\left(\Gamma_{\alpha}(p)\right) \\
\Gamma_{0}(p) & =p
\end{aligned}
$$

defines a group of homeomorphism $\Gamma_{\alpha}: \mathbf{R}^{n_{\longmapsto}} \mapsto \mathbf{R}^{n}$ and the following group of unitary transformations on $L^{2}\left(\mathbf{R}^{n}, d^{n} p\right)$

$$
\left(U_{\alpha} \Psi\right)(p)=\left|\operatorname{det}\left(\frac{\partial \Gamma_{\alpha}^{i}}{\partial p_{j}}(p)\right)\right|^{1 / 2} \Psi\left(\Gamma_{\alpha}(p)\right)
$$

we then have

$$
\begin{aligned}
\frac{d}{d \alpha}\left(U_{\alpha} \Psi\right)_{\alpha=0}(p) & =\sum_{i} g_{i}(p) \frac{\partial \Psi}{\partial p_{i}}(p)+\frac{1}{2} \sum_{i=1}^{n} \frac{\partial g_{i}}{\partial p_{i}}(p) \cdot \Psi(P) \\
& =-i(A \Psi)(p),
\end{aligned}
$$

where $A$ is the self-adjoint extension of $\hat{A}$.

Let us finally note that $D(A)$ contains $D(|x|)$. 


\section{Appendix II}

Proposition II.6. Let $H, A$ be operators that satisfy conditions (a)...(d). Then:

1. Let $g$ be any function with $t \hat{g}(t) \in L^{1}(\mathbf{R}, d t)$, then

$$
g(H): D(A) \cap D(H) \rightarrow D(A) .
$$

2. Let $B^{*} B=P_{H} i[H, A]^{0} P_{H}$ as defined in the lemma of Sect. II. Then $\left[B^{*} B, A\right]$ is a bounded map from $\mathscr{H}_{+2}$ into $\mathscr{H}_{-2}$.

3. $G_{z}(\varepsilon): D(A) \rightarrow D(A) \cap D(H)$.

Proof. Let $\Psi \in D(A) \cap D(H), A(\lambda)=A i \lambda(A+i \lambda)^{-1}$ for some sufficiently large $|\lambda|$. Then

$$
\left\|\left\{A(\lambda) e^{-i H t}-e^{-i H t} A(\lambda)\right\} \Psi\right\| \leqq \sup _{\substack{\Phi \in D(H) \\\|\Phi\|=1}} \int_{0}^{t}\left(\Phi \mid e^{+i(s-t) H}[H, A(\lambda)] e^{-i s H} \Psi\right) d s \mid .
$$

Since $e^{-i H s}$ leaves $D(H)$, and also $A(\lambda)$ by Proposition II.3, we then have

$$
\left\|\left\{A(\lambda) e^{-i H t}-e^{-i H t} A(\lambda)\right\} \Psi\right\| \leqq|t| \sup _{|s| \leqq|t|} \sup _{\substack{\Phi^{\prime} \in D(A) \cap D(H) \\\left\|\Phi^{\prime}\right\|=1}}\left|\left(\Phi^{\prime} \mid[H, A(\lambda)] e^{-i s H} \Psi\right)\right| .
$$

By Eq. (II.3) in Propositions II.4 and II.3, one then sees that

$$
\begin{aligned}
\left\|A e^{-i H t} \Psi\right\| & \leqq \lim _{|\lambda| \rightarrow \infty}\left\|A(\lambda) e^{-i H t} \Psi\right\| \\
& \leqq c|t|\|(H+i) \Psi\|+\|A \Psi\|
\end{aligned}
$$

It is now enough to use the identity $g(H)=\int_{-\infty}^{+\infty} \hat{g}(t) e^{-i H t} d t$ to see that

$$
g(H): D(A) \cap D(H) \rightarrow D(A) \quad \text { if } \quad|t| \hat{g}(t) \in L^{1}(\mathbf{R}, d t)
$$

and that

$$
\|\{A g(H)-g(H) A\} \Psi\| \leqq c\|(H+i) \Psi\| \int_{-\infty}^{+\infty}|t||\hat{g}(t)| d t .
$$

Let $B^{*} B=P_{H} i[H, A]^{0} P_{H}$. Since $P(\lambda)$ is smooth, its Fourier transform decays rapidly. Hence $P_{H}$ takes $D(A) \cap D(H)$ into $D(A) \cap D(H)$ and so $\left[B^{*} B, A\right]$ in the sense of quadratic forms on $D(A) \cap D(H)$ can be written:

$$
\left[B^{*} B, A\right]=\left[P_{H}, A\right][H, A]^{0} P_{H}+P_{H}\left[[H, A]^{0}, A\right] P_{H}+P_{H}[H, A]^{0}\left[P_{H}, A\right] .
$$

By hypothesis (d) and the relation (II.9), the form $\left[B^{*} B, A\right]$ on $D(A) \cap D(H)$ is bounded as a map from $\mathscr{H}_{+2}$ into $\mathscr{H}_{-2}$ and in particular if

$$
\begin{aligned}
& \Psi \in D(H)\left\|\left[\left(H-z-i \varepsilon B^{*} B\right), A(\lambda)\right] \Psi\right\|_{-2} \\
& \leqq \sup _{\substack{\Phi \in D(A) \cap D(H) \\
\|\Phi\|_{+2}=1}}\left\{\left|\left(\Phi \mid\left[H-z-i \varepsilon B^{*} B, A\right] i \lambda(A+i \lambda)^{-1} \Psi\right)\right|\right. \\
& \left.\quad+\left|\left(\Phi \mid A(A+i \lambda)^{-1}\left[H-z-i \varepsilon B^{*} B, A\right] i \lambda(A+i \lambda)^{-1} \Psi\right)\right|\right\} .
\end{aligned}
$$


By Proposition II.3, the operators $\lambda(A+i \lambda)^{-1}$ and $A(A+i \lambda)^{-1}=1-i \lambda(A+i \lambda)^{-1}$ are uniformly bounded from $\mathscr{H}_{+2}$ into $\mathscr{H}_{+2}$ for $\lambda$ large enough. It follows that $\left[H-z-i \varepsilon B^{*} B, A(\lambda)\right]$ are uniformly bounded (in $\lambda$ ) from $\mathscr{H}_{+2}$ into $\mathscr{H}_{-2}$. It follows that $G_{z}(\varepsilon)=\left(H-z-i \varepsilon B^{*} B\right)^{-1}$ preserves $D(A)$ and hence :

$$
G_{z}(\varepsilon): D(A) \rightarrow D(A) \cap D(H) .
$$

Acknowledgements. I am grateful to P. Perry for his careful reading of the French version of this work and for his translation, and to B. Simon for encouraging its publication.

\section{References}

1. Kato, T.: Perturbation theory for linear operators. Berlin, Heidelberg, New York: Springer 1966

2. Agmon, S.: Ann. Scuola Norm. Sup. Pisa, Ser. 4, 2, 151-218 (1975)

3. Aguilar, J., Combes, J.M.: Commun. Math. Phys. 22, 269-279 (1971)

Balslev, E., Combes, J.M.: Commun. Math. Phys. 22, 280-294 (1971)

4. Reed, M., Simon, B.: Methods of modern mathematical physics. Tomes II and III. New York: Academic Press 1979

5. Enss, V.: Commun. Math. Phys. 61, 285 (1978)

6. Simon, B. : Duke Math. J. 46, 119-168 (1979)

Communicated by B. Simon

Received March 19, 1980; in revised form July 21, 1980 